\title{
Stability Control of Renewable Energy Sources in Distribution Systems
}

\author{
Josef Tlusty \\ Czech Technical University, FEE \\ Prague, Czech Republic
}

\author{
Zdenek Muller \\ Czech Technical University, FEE \\ Prague, Czech Republic
}

\author{
Viktor Valouch \\ Academy of Sciences of the CR \\ Prague, Czech Republic
}

\begin{abstract}
Deregulation in the power market has encouraged the move towards distributed generation, where many smaller generating plants located close to major loads are penetrating into interconnected power systems. When the penetration level increases, several other problems will need to be faced and solved. Stability problems may arise, new planning tools will be needed, dispatching of energy sources with typical random production will be necessary, protection systems must be integrated and revised. In order to simulate power system dynamics programmable opened software in Matlab - DynaSolv was developed. The paper describes various principles of penetration synchronous machine type DGS into distribution network. This study provides possible complex analysis view on DGS stability issues, which can answer the possibility of connection.
\end{abstract}

Index terms- Stability, Distributed generation, Globally penetrated system, Eigenvalues.

\section{INTRODUCTION}

Nowadays, generating electricity using decentralized generators of relatively small scale is attracting a great interest from electric energy researchers. Such kind of generation is known as Distributed Generation Systems (DGS).

Main aim of using large amount of DGS in the future is to increase system reliability and lower the cost of power through the use of on-site generation. Since the DGS Penetration Level (PL) is still low (DG power generated less than $20 \%$ of power consumption in a sub-grid), the technical problems related to DG are limited to voltage profile support and control of interfacing conversion devices. When PL increases, several other problems will need to be faced and solved.

\section{A. Distributed Generation}

Distributed Generation Systems (DGS) can provide energy solutions for some customers that are most cost-effective, environmentally friendly or provide better quality or reliability than conventional sources. DGS can be defined as not centrally planned sources, which operate on distribution network voltage level (LV or MV) and their one-unit power output is $5 \mathrm{~kW}-5 \mathrm{MW}$ (according CIGRE definition less than $50 \mathrm{MW}$ ).

Within new projects in the future in supplying local areas, a Power Operation and Power Quality Management System (POPQMS) must be developed. POPQMS will be a communication infrastructure and optimization tool for distribution grids which is expected to perform power quality monitoring and control and to optimize grid operation through distributed generation control and demand side load management.

\section{B. Power Operation and Power Quality Management System}

In synthesis POPQMS is expected to collect information about topology situation and power quality in the network segments under control, provide local strategies for power quality and security improvement, observes and learns patterns of generation and consumption, including (over) load of grid infrastructure, generates schedules for economically (cost) and technically (power quality) optimized operation of controllable generators and loads, predict times with unbalance of generation and consumption of the grid segment and develop reaction strategies, for medium and long term optimization reacts on local under- or over-voltage by increasing or decreasing generation or consumption accordingly for both active power and reactive power, for short term reaction and intervention develop adequate reaction patterns for likely events, to be executed upon necessity, and instructs distributed components about their specific optimized reaction modes

\section{MULTIMACHINE SYSTEM MODEL}

The power system can be in general described by a set of DAE:

$$
\left[\begin{array}{l}
\dot{x} \\
0
\end{array}\right]=\left[\begin{array}{l}
f(x, y, u, t) \\
h(x, y, u, t)
\end{array}\right]
$$

where $t$ represents time, $\boldsymbol{y}$ vector of algebraic variables, $\boldsymbol{u}$ vector of system parameters and $\boldsymbol{x}$ vector of state variables. Interconnected multimachine system thus generates:

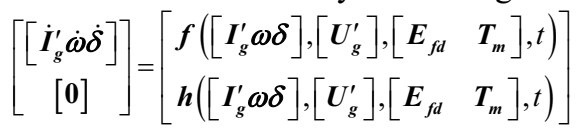

where $\left[\boldsymbol{I}_{g}^{\prime} \boldsymbol{\omega} \boldsymbol{\delta}\right]$ is state variable vector

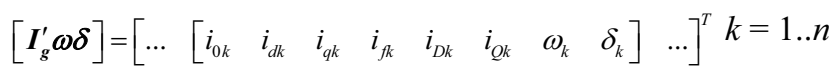


and $\left[\boldsymbol{U}_{g}^{\prime}\right]=\left[\begin{array}{llll}\ldots & u_{d k} & u_{q k} & \ldots\end{array}\right] \quad k=1 \ldots n$ represents voltage vector of algebraic variables taking $n$-machine system under consideration. Machines nodes' interconnection is described by reduced admittance resp. impedance matrices:

$$
\begin{aligned}
{\left[\mathbf{Y}_{R E D}\right] } & =\left[\begin{array}{lll}
g_{11}+j b_{11} & & \\
& \ldots & \\
& & g_{g g}+j b_{g g}
\end{array}\right], \\
{\left[\mathbf{Z}_{R E D}\right] } & =\left[\begin{array}{lll}
r_{11}+j x_{11} & & \\
& \ldots & \\
& & r_{g g}+j x_{g g}
\end{array}\right]
\end{aligned}
$$

Assuming matrix $[\mathbf{M}]=[\mathbf{T} *] .\left[\mathbf{Y}_{R E D}\right][\mathbf{T}] \quad$ respectively $[\mathbf{M}]^{-1}=[\mathbf{T} *] .\left[\mathbf{Z}_{R E D}\right] .[\mathbf{T}] \quad$ expressing machines nodes' interconnection respecting angular displacements $\theta_{1}, \theta_{2} \ldots, \theta_{n}$ of machines' $q_{1}, q_{2} \ldots, q_{n}$ axes towards reference $Q$ axis, the machine voltages can be expressed as

$$
\left[\boldsymbol{U}_{g}^{\prime}\right]=[\mathbf{T} *] \cdot\left[\mathbf{Z}_{R E D}\right] \cdot[\mathbf{T}] \cdot\left[\boldsymbol{I}_{g}^{\prime}\right]=[\mathbf{M}]^{-1} \cdot\left[\boldsymbol{I}_{g}^{\prime}\right]
$$

From the algebraic part voltage vector $\left[\boldsymbol{U}_{g}^{\prime}\right]$ could be thus obtained as

$$
\left[\boldsymbol{U}_{g}^{\prime}\right]=\boldsymbol{h}_{U}^{-1}\left(\left[\boldsymbol{I}_{g}^{\prime} \boldsymbol{\omega} \delta\right], t\right)
$$

which substituted to first part of (1) gives

$$
\left[\dot{I}_{g}^{\prime} \dot{\boldsymbol{\omega}} \dot{\delta}\right]=\boldsymbol{f}\left(\left[\boldsymbol{I}_{g}^{\prime} \boldsymbol{\omega} \boldsymbol{\delta}\right], \boldsymbol{h}_{U}^{-1}\left(\left[\boldsymbol{I}_{g}^{\prime} \boldsymbol{\omega} \boldsymbol{\delta}\right], t\right),\left[\begin{array}{ll}
\boldsymbol{E}_{f d} & \boldsymbol{T}_{m}
\end{array}\right], t\right)=\boldsymbol{f}^{\prime}\left(\left[\boldsymbol{I}_{g}^{\prime} \boldsymbol{\omega} \boldsymbol{\delta}\right],\left[\begin{array}{ll}
\boldsymbol{E}_{f d} & \boldsymbol{T}_{m}
\end{array}\right], t\right)
$$

The equation (7) transfers original DAE problem (2) to ODE problem for interconnected power system. If additionally the system (7) is time invariant

$$
\boldsymbol{f}^{\prime}\left(\left[\boldsymbol{I}_{g}^{\prime} \omega \delta\right],\left[\begin{array}{ll}
\boldsymbol{E}_{f d} & \boldsymbol{T}_{\boldsymbol{m}}
\end{array}\right], t\right)=\boldsymbol{f}^{\prime}\left(\left[\boldsymbol{I}_{g}^{\prime} \omega \delta\right]\right)
$$

The function $\boldsymbol{f}^{\prime}$ is generally symbolic for $n$-machine system. This fact is important for further analysis.

\section{PRINCIPAL VIEW ON POWER SYSTEM DYNAMIC STABILITY}

According the definition of dynamic stability described in [6], for the dynamic stability analysis a system linearization at fixed point can be used. Many criteria can be formulated with help of eigenvalue analysis. Assume nonlinear system (1). Its linearization at fixed point is expressed as

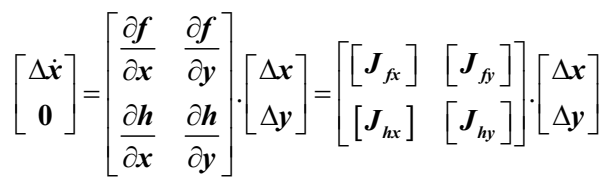

can be simplified to formula

$$
[\Delta \dot{x}]=\left(\left[J_{f x}\right]-\left[J_{f y}\right] \cdot\left[J_{h y}\right]^{-1} \cdot\left[J_{h x}\right]\right) \cdot \Delta x=\left[J_{R E D}\right] \cdot \Delta x
$$

where $[\boldsymbol{J}]$ are Jacobian sub-matrices and $\left[\boldsymbol{J}_{\boldsymbol{R E D}}\right]$ reduced system Jacobian matrix, which can be also obtained as $\left[\boldsymbol{J}_{\boldsymbol{R E D} D}\right]=\left[\frac{\partial \boldsymbol{f}^{\prime}}{\partial \boldsymbol{x}}\right]$ from (7) and (8).

Eigenvalue analysis is based on principles linearization of nonlinear system at fixed point. System is thus (dynamic) stable if all the eigenvalues are non-positive. Stability boundary is a set of equilibrium points, where at least one analyzed system parameter differential change invokes unstable equilibrium point. In other words, stability boundary is a set of points of system bifurcation. Various methods can be used to find out stability boundary and identify, which kind of instability appears, if the boundary is reached. In general, two kinds of bifurcations on stability boundary may occur. Third kind of bifurcation described below does not strictly imply system instability, however causes difficulty of identifying stable region. Identification of type of bifurcation according angular criterion $\mathrm{DSI}_{2}$ is shown in Fig. 1 .

Saddle Node Bifurcation (SNB) is a (steady state) point on stability boundary where a pair of equilibrium points meets and disappears with zero eigenvalue of system Jacobian matrix. One point is thus unstable - saddle, hence the other one stable - node.

Hopf Bifurcation (HB) occurs, if a pair of complex conjugated eigenvalues of system Jacobian matrix crosses the imaginary axis. At a Hopf Bifurcation system becomes unstable interacting with limit cycle and passes to unstable oscillations.

Following Dynamic Stability Indicator $\left(\mathrm{DSI}_{1}\right)$ can be used for simple identification whether system is stable or not:

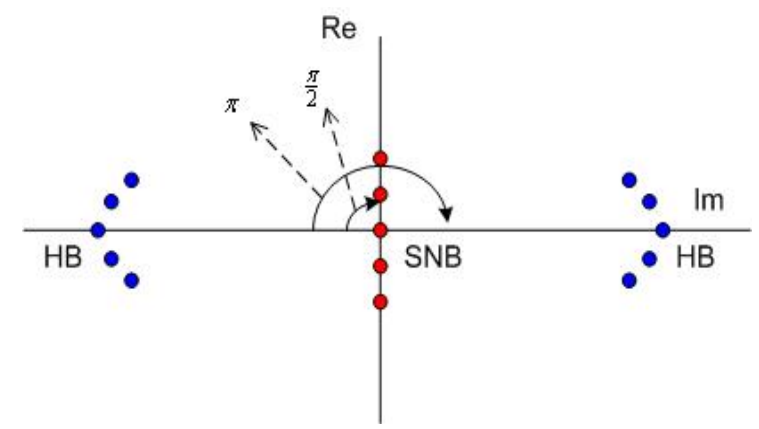

Fig. 1: Identification of type of bifurcation acording angular criterion $\mathrm{DSI}_{2}$

$$
\boldsymbol{D S I}_{1}=\max [\operatorname{Re}[\lambda]]
$$

$\mathrm{DSI}_{1}$ implies the "quality of stability" i.e. rate of system ability to return to stable equilibrium point respectively rate of system ability to leave unstable equilibrium point. However, from $\mathrm{DSI}_{1}$ can't be identified SNB and HB points. Better way to identify stability boundary is complex plane angular criterion $-\mathrm{DSI}_{2}$. 
$\mathbf{D S I}_{2}=\max [\operatorname{Angle}[j . \lambda]]=\max \left[\operatorname{arctg}\left(\frac{\operatorname{Re}[\lambda]}{\operatorname{Im}[\lambda]}\right)\right]$

$\mathrm{DSI}_{2}$ can be also used for identifying SNB and HB points. $\mathrm{DSI}_{2}$ uses the fact, that system at HB point has always two complex conjugated eigenvalues. It can be easily proven, that at $\mathrm{HB}$ point $\mathrm{DSI}_{2}$ unstable point is equal to $\pi$, hence at $\mathrm{SNB}$ point $\mathrm{DSI}_{2}$ to $\frac{\pi}{2}$.

Except from eigenvalue analysis, singular value analysis [1], [3], [4] is being used. The method is based on singular value decomposition of reduced Jacobian matrix (10). The minimal value of singular number $\sigma_{\min }\left(\left[J_{R E D}\right]\right.$ ) expresses proximity to singular point (i.e. $\mathrm{HB}$ or SNB). If $\sigma_{\min }$ has zero value, SNB or HB is reached and stability boundary is thus detected.

Dynamic stability analysis is demonstrated on following examples for better understand of its use.

\section{Single MACHINE TO INFINITE BUS -DYNAMIC STABIULITY ANALYSIS}

Dynamic stability eigenvalue analysis can be demonstrated on simple case study "Single Machine to Infinite Bus" (SMIB). Infinite bus operates at nominal voltage, system load is $10 \mathrm{MW}$. Stability is analyzed for two parameters of $13 \mathrm{MW}$ unregulated synchronous machine - active and reactive power. Results are presented in following figures $2-5$.

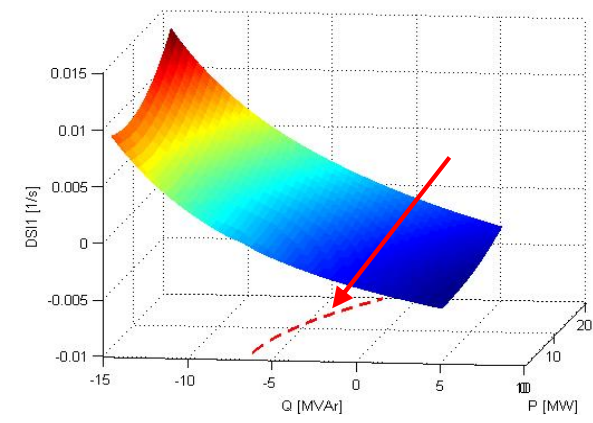

Fig. 2: Eigenvalue analysis of SMIB (DSI $)$, detail of stability boundary - "underexcitation limit"

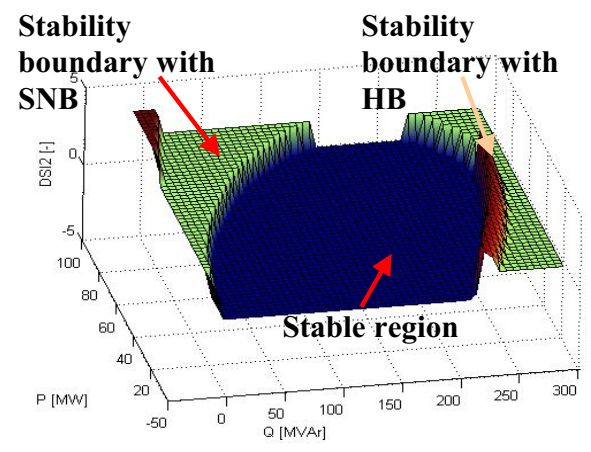

Fig. 3: Eigenvalue analysis of SMIB (DSI $)$, entire stable region with voltage stability limit

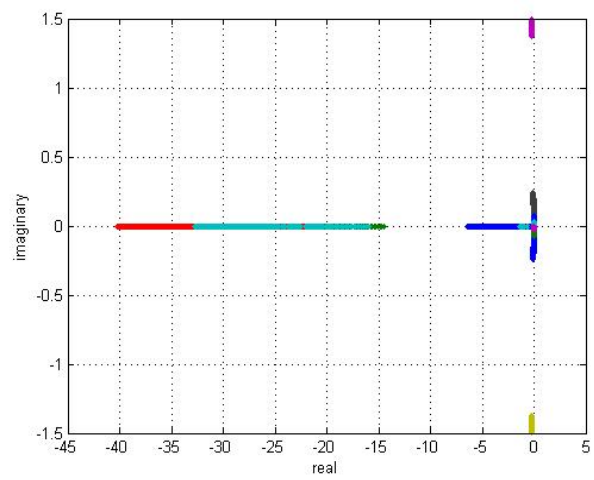

Fig.4: SMIB system eigenvalues at $\mathrm{P}=0$

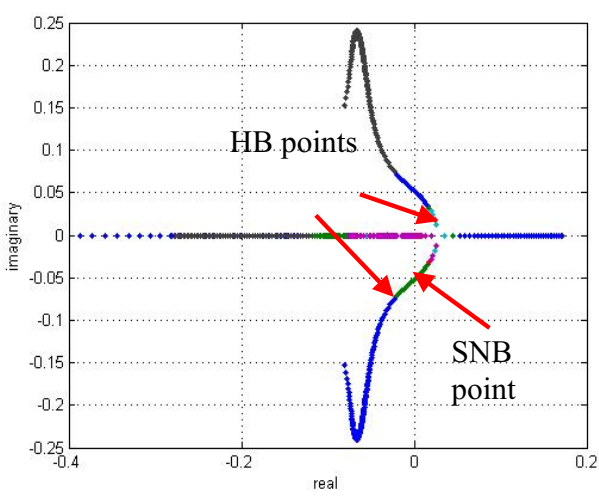

Fig. 5: Detail of SMIB system eigenvalues at $\mathrm{P}=0 \mathrm{MW}$

Making conclusions to the SMIB case study, it must be noted, that only region of stability was subject of the study. Real operating region is certainly constrained by voltage, current and machine thermal limits. However, eigenvalue analysis gives the sufficient results of machine-network system capability. Furthermore, stability boundary is precisely detected as well. Specific parts of the results correspond to conventionally determined under excitation limits.

\section{NODE DISTRIBUTION NETWORK EXAMPL}

Presented tools for DGS power system analysis are demonstrated on distribution network model. The network modeled in MATLAB (such as the illustrative examples from introduction of this chapter) contains 38 passive PQ loads at MV level $22 \mathrm{kV}, 4 \mathrm{HV} 110 \mathrm{kV}$ substations and one EHV 400 $\mathrm{kV}$ slack node, which represents transmission system. Branch parameters chosen in the model are typical parameters of Czech distribution network. It can be noted, that this network represents an example of supplying a town with 50 thousand inhabitants and $30 \mathrm{~km}$ surroundings including suburbs and municipal supplies.

Main aim of this study is to disclose this system behavior beyond high level of DGS penetration by sources with SM generators. For this purpose various types of SM generators (salient pole, round), two types of connection to distribution network. 
Due to system complexity primary $0 d q$ model was analyzed.

Two main principles of DGS penetration were studied. As first is presented locally penetrated system, hence as second case is presented globally penetrated system. In both cases, sources are connected one by one to random nodes of affected area.

\section{Globally Penetrated system}

For globally penetrated system were taken same analysis principles as in the case of locally penetrated systems under consideration. DGS sources connection sequencing principle is applied to whole network MV nodes.

\section{Dynamic stability analysis}

As in previous example, for the first part of study uniform source types were chosen. Following figures are depicting only one source type, because same conclusions considering operable regions dependence on source types can be claimed. Hence the best option of uniform source (salient-pole machine example connected via $6.3 / 22 \mathrm{kV}$ transformer) was chosen.

The results of study imply that for $5^{\text {th }}$ connected DGS source can't be found any relevant operational point, which satisfies dynamic stability (note, that other connected machines are assumed as PU nodes in their connection points). To obtain a suitable steady state solution, new regulation principles must be applied. In further text is purposed simple regulation to target steady state values proportional regulation of reactive powers (existing similar systems in centralized generation is called ASRU). Steady state of other connected machines from $n=5$ DGS sources is thus computed for constant power factor (purposed target values for proportional regulation). Following fig.6 and 7 show the penetration level up to 13 connected DGS sources i.e. $13 \times 2.5=32.5 \mathrm{MW}, \mathrm{PL}=35.7 \%$ (note, that 13 sources doesn't mean $P L$ limitation). However, to satisfy voltage limits $\pm 10 \%$, distribution transformers' ratios with increasing $P L$ have to be updated.

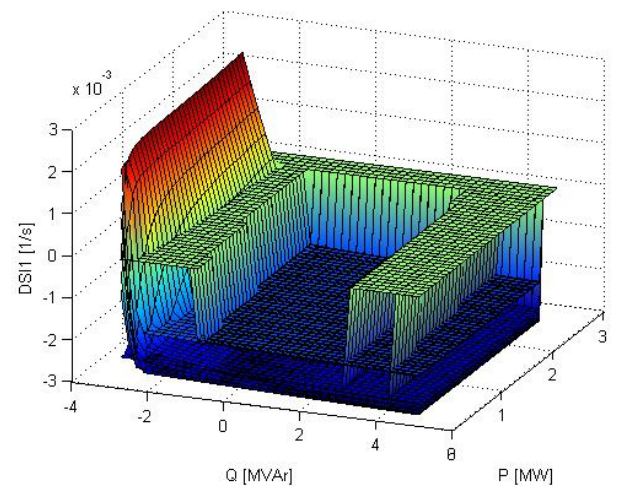

Fig. 6: DSI $_{1}$ after 5-13 DGS sources connection (salient-pole 2.5 MW machines, type A connection) with constant power factor principle

Same type of analysis not presented here proves ability of connection $13 \times 6 \mathrm{MW}$ sources (i.e. $P L=85 \%$ ). Other studies also prove connectibility of random types of machines with application constant power factor principles.

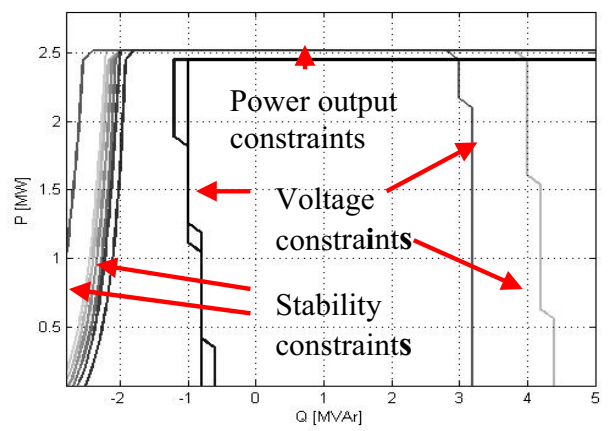

Fig. 7: Operable regions after 5-13 DGS sources connection (salient-pole $2.5 \mathrm{MW}$ machines, type A connection) with constant power factor principle

Finally, following three figures show system eigenvalues for one, 13 uniform and 13 random machines connected to considered distribution network.
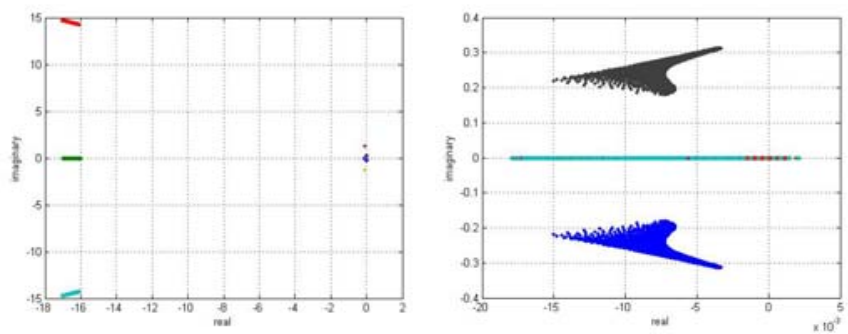

Fig. 8: Oscillatory modes for DGS, eigenvalues spectrum for one 2.5 MW source
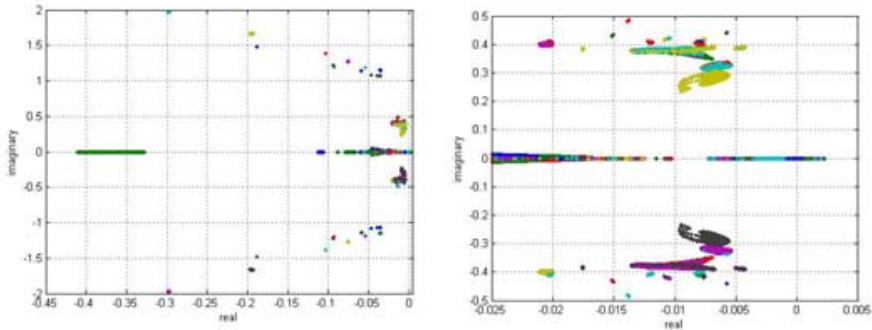

Fig. 9: Oscillatory modes for DGS, eigenvalues spectrum for 13 random sources
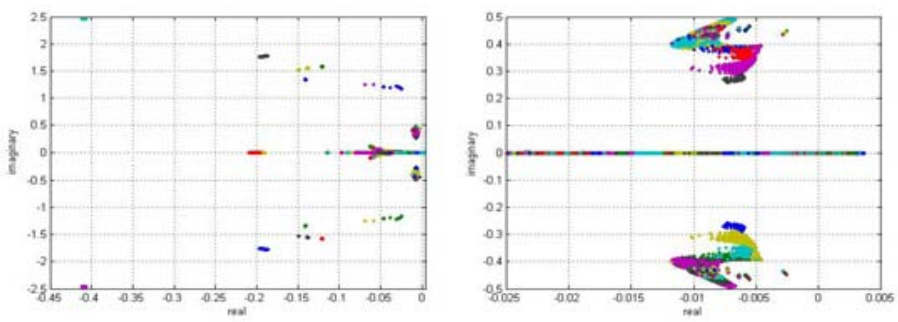

Fig. 10: Oscillatory modes for DGS, eigenvalues spectrum for 13 uniform $6 \mathrm{MW}$ sources

Comparison of fig. 9 and 10 provides information about system oscillatory modes. System with uniform machines contains three specific frequencies around $1 \mathrm{~Hz}$, hence the system with randomly chosen machines contains in this range many oscillatory modes. This fact could be important for 
further possible subsynchronous resonance analysis and DGS stabilizer designing.

\section{Transient stability analysis}

Following figures show voltages at selected $22 \mathrm{kV}$ nodes. Same as in locally penetrated system, 4 fault nodes with clearing time $200 \mathrm{~ms}$ is applied for transient stability analysis. Tested cases respect the results from dynamic stability ana

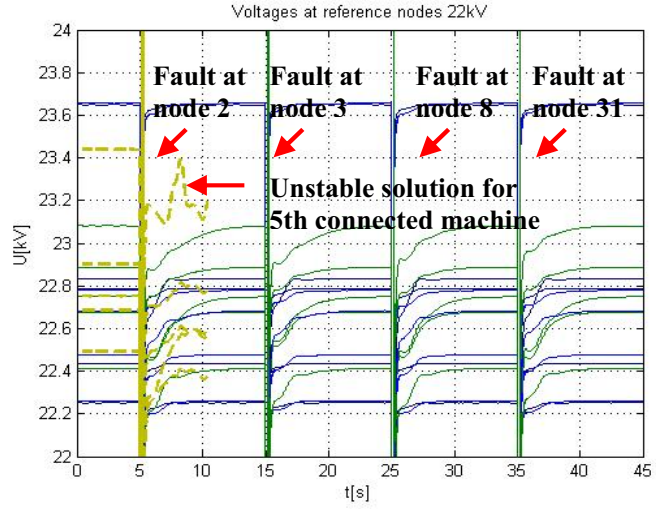

Fig. 11: Fault voltages at selected nodes (salient-pole $2.5 \mathrm{MW}$ unregulated machines, type A connection)

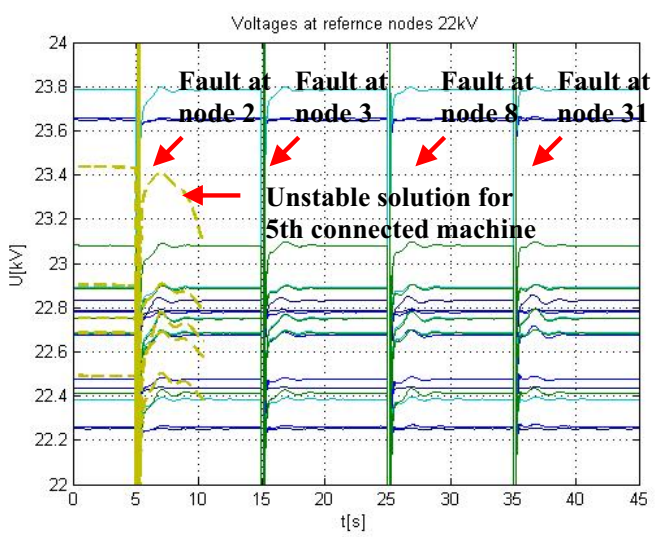

Fig. 12: Fault voltages at selected nodes (salient-pole 2.5 MW regulated machines, type A connection)

Fig. 11 and 12 show thus transients from system steady state without constant power factor principle. Solutions are stable except from $5^{\text {th }}$ connected machine (note, that this case was excluded in dynamic stability analysis).

\section{CONCLUSION}

The paper describes various principles of penetration synchronous machine type DGS into distribution network. The study was aimed to possible operation area of the connected sources with regard to stable operation in steady state conditions and stable operation after clearing the fault. Note, that other important issues for successful DGS installing process were not discussed within this study (such as necessary increase of short circuit level, protection issues, peaking and premium power delivery issues, reliability issues and also some special cases of transients like pass into islanded operation state). This study however provides possible complex analysis view on DGS stability issues, which can answer the possibility of connection. From this point of view, following results are obtained.

It can be assumed, that in locally penetrated system (i.e. to one feeder) inter-machine couplings are smooth and voltages are similar and thus such a system's behavior is stable and thus doesn't strictly require centralized regulation. It is shown, that local penetration (which means centralized generation inside distribution network) could be possible low cost way, how to increase PL of distribution system with strongly restricted advantages of DGS use.

Globally penetrated system requires from very small amount of installed DGS new centralized control strategies to maintain stable operation. Except from using new control strategies with target steady state values, new phenomena such as subsynchronous resonance may arise and have to be resolved. New stabilizers must be developed to avoid danger oscillations, which may be caused on both demand and generation side.

Finally, it must be noted, that stable operation at same PL of DGS strongly depends on chosen technology (i.e. machine type). From that point of view, preferred technologies must be determined.

\section{ACKNOWLEDGEMENT}

The financial supports of the Grant Agency of the Academy of Sciences of the Czech Republic (project No. IAA 200760703) and of the Ministry of Education, Youth and Sports (Reasearch Plan MSM 68404770017 of the Czech Technical University) are highly acknowledged.

\section{REFERENCES}

[1] S. Ayasun, Ch. O. Nwankpa, H. G. Kwatny: Computation of Singular and Singularity Induced Bifurcation Points of Differential Algebraic Power System Model, IEEE Paper, Vol. 51, No. 8, 2004

[2] M. A. Pai: Power System Stability by Lyapunov's Method, N Holland Publishing Company, 1981

[3] L. Qi: AC Systems Stability Analysis and Assessment for Shipboard Power Systems, Texas A\&M University, doctoral thesis, 2004

[4] S. Repo: On-Line Voltage Stability Assessment of Power System an Approach of Black-Box Modeling, Tampere, doctoral thesis, 2001

[5] G. Teschl: Ordinary Differential Equations and Dynamical Systems, Universität Wien, 2004

[6] N.Rouche, P.Habets, M.Laloi: "Stability Theory by Liapunov's Direct Method", Springer-Verlag, 1997 